\title{
A unified Method for assessing the Observability of Dynamic Complex Systems in Biology
}

\author{
Juan G. Diaz Ochoa ${ }^{1 *}$ \\ PerMediQ GmbH \\ Salzbergweg 18 D-85368 Wang \\ Germany
}

21.01.2022

Key Words: Persistent Topology; Time Series; Geometric Information Theory; Persistent Entropy; Causality; Observability; Dynamic Complex Systems

\section{ABSTRACT}

\section{Problem:}

Systems biology is essentially based on the assumption that the complexity of a system can be described by almost generic models to predict the behavior of many other similar systems. To this end, inductive (data-intensive) or deductive (mechanistic) models are currently being developed either to discover patterns and identify plausible correlations from past events, or to connect different causal relationships of interacting elements to construct predictive models. The use of any mathematical approach, or combination of mathematical approaches, presupposes the existence of constant and observable universal causal principles for all biological systems. However, there are currently no tools to assess the robustness of adoption over these universal causal principles, even considering that organisms not only respond to inherent processes and environmental stimuli across multiple scales, but also integrate information across and within these scales, introducing a degree of uncontrollable uncertainty.

\section{Methodology:}

To this end, we have developed a method to better evaluate these causal processes by evaluating the information contained in the dynamic trajectories, using concepts of geometric information theory and persistent homology to analyze patterns in time series, so that recognizing the persistence of these patterns over different time periods leads to the evaluation of stable causal relationships. Since we are assessing causal relationships from the patterns in the time series, we are here going beyond mere feature engineering. With this measure, and together with the evaluation of persistent entropy in the trajectories in relation to different individual systems, we have developed a method called the $\Phi$ - $S$ Diagram as a measure of complexity to better recognize when organisms follow mechanistic pathways

\footnotetext{
${ }^{1}$ Corresponding author: juan.diaz@permediq.de; https://orcid.org/0000-0002-9893-4068
} 
or respond autonomously and individually, which is a limitation for both the inductive and deductive modeling methods.

\section{Results:}

We calculated the $\Phi$-S Diagram using a deterministic data set available in the UCl repository for reference to test the interpretability of the method, as well as health data available in the same repository that includes the physiological response to movement measured in individuals outside of laboratory conditions. We were able to confirm the mechanistic character in both data sets. Although the current data show that the cardiac response to exercise could be explained in a mechanistic way, we have also discovered that some individuals have a more autonomous than aleatoric response, implying that there are persistent individual variations that limit the universal observability of the system, suggesting that such individuals need an empirical rather than a systemic approach. This proof of concept presents a step towards a clearer framework for the theoretical representation of complex biological systems.

\section{INTRODUCTION}

The core idea of systems biology consist of reducing complex systems into clear interconnected elements, leading into either white box and deductive models, integrating causal paths from data obtained from system's relevant physical-chemical interactions, or black box models, based on the automatic integration of large amounts of information using for instance neuronal networks to recognize relevant system's patterns (Zitnik et al., 2019) (Baker et al., 2018). The hope behind this effort is to get insights into the function, effects of interventions/perturbations and future development of the bio-system. Essentially such approaches consider living systems just as an emergent phenomenon of all these interconnected phenomena (Kostić et al., 2020).

These systemic approaches are fundamental in the definition of systems biology and systems medicine, which is a framework primarily concerned with the mathematical description of functional aspects of biological systems (Green, 2021) ${ }^{2}$ (Boogerd et al., 2007). The success of these systemic approaches has a clear pragmatic advantage, as they guide the identification and understanding of fundamental biological mechanisms that can be described using mathematical models useful in biotechnology and biomedicine for the development of, for example, synthetic organisms, efficient bioprocesses, drugs or therapies (Alon, 2006). Often, identifying these models is a great effort, not only in defining the appropriate modelling scale, equations, and the set of parameters that both represent biological processes in deductive models, but also for the amount of information required to develop high-quality inductive models. In both cases, mechanisms or correlations assume an inherent constant behavior that could be reduced to simple first principles.

But this kind of approaches often ignore the fact that coarse-grained physical systems can be much more effective in terms of intrinsic cause-effect power than conventional micro level systems. According to this, while mechanisms are usually characterized in causal terms, it is not the case that every cause act through or is a part of some mechanism, which is understood as a more or less complex arrangement of causal factors that are productive of change ${ }^{3}$ (Reiss and Ankeny, 2016). Living systems, which are characterized by this coarse-grained character, behave as unitary 'whole' from their own intrinsic perspective in order to maintain homeostatic states, implying that living systems are much

\footnotetext{
${ }^{2}$ https://plato.stanford.edu/entries/systems-synthetic-biology/\#BiolDigiAge
}

${ }^{3}$ https://plato.stanford.edu/entries/medicine/\#IntrHowShouWeDefiHealDise 
more than mere "systems" with mechanistic responses, since they are autonomous (from a coarse grained perspective), able of integrating self-defined and self-maintained borders, implying some notion of intrinsic causal control (Marshall et al., 2017). Therefore, it is necessary to realize the uniqueness of certain basic principles of biology that are not applicable to the inanimate world. ${ }^{4}$ This notion could encompass the idea that biological systems are non-trivially reducible to physical laws (Walker, 2019).

The main problem of this autonomy is that the unitary whole generating this unity must account the intervention of different elements across several scales, limiting our ability as observers to make objective observations as well as clear definitions about which are the appropriate coarse graining required to correctly describe a system. This whole can be characterized by the integrated information of the system, a method that has been recently used to investigate the relationship between this integrated information and the fitness of evolving systems (Joshi et al., 2013a).

This autonomy could imply that models in systems biology have a limited applicability, a concern that has been shared by many scientists and scholars, i.e., the possibility that biological systems are nonreducible into Hamiltonian functions (Walker, 2019). Instead, biology is characterized by the persistent absence of universal principles and a branched causation, which would be amenable to derive consistent and rigorous descriptions of biological systems, similar to physics or even chemistry (Ellis and Kopel, 2019). Furthermore, this autonomy could imply that these systems are themselves observers that not only interact with the environment, but also may simultaneously observe any external experimenter aiming to get objective observations of these systems ${ }^{5}$. Indeed, the evaluation of the degree of autonomy of a complex living system is relevant to assess its observability ${ }^{6}$. This fact makes under some circumstances the parameter identification and or establishment of plausible correlations not only a very difficult but, de facto, an impossible task.

Thus, considering a network-like perspective (commonly used in systems biology), we are not simply dealing with network models with evolving links, but in general with network models whose nodes (representing internal states) and links (representing the couplings between these states) change in an autonomous and rather holistic way, depending on the current scale of observation, which is defined by the system itself (Diaz Ochoa, 2020). We are dealing with a kind of autonomy that is not reducible to mere intelligence (Koch, 2019), implying systems that are much more complex than evolving networks: the representation of the nodes and the links is currently a coarse-grained state that is accessed from outside, but which is defined by the system itself. In a nutshell, we are dealing with systems owning an irreparable inherent uncertainty. The current paradigms in mathematical modelling mainly focus on the definition of models aiming to predict future outcomes. But in recent years alternative approaches investigate the development of models that focus more on the assessment of the persistent uncertainty of the systems. Thus, 'models to predict and control the future' are replaced by 'models to map our ignorance about the future' (Ravetz, 2003).

Our goal is to exactly evaluate causal paths in complex systems and its contribution in the model identification (Baker et al., 2018), as well as to assess the degree of autonomy of a complex-biological system and in this way map our own degree of ignorance or observability of this system, i.e. our

\footnotetext{
${ }^{4}$ https://www.cambridge.org/core/books/what-makes-biology-unique/autonomy-ofbiology/B59719F0B2A417A7C220AD17443BC796

${ }^{5}$ This situation resembles the uncertainty principle in quantum mechanics.

${ }^{6}$ Which is completely different to the concept of autonomy in engineering. See e.g. https://en.wikipedia.org/wiki/Autonomous system (Internet)
} 
capacity to identify models, accounting in this way the degree of ignorance about the system's future. Indeed, we are departing from the fact that the observations -input data- contains inherent imperfections generated by the organism's autonomy, regardless the quality of the sampled data. To this end it is important to analyze and quantify the degree of causal relationships between different elements in the system (Oizumi et al., 2016), as well as the potential persistent structures in the data leading to relative individual differences regarding a population of organisms. We combine this analysis and complexity measures in a diagram to quantify how feasible is to employ modeling paradigms, like network models defined as canvas for the representation of interconnected elements?

The novelty of our approach is the combination of methods to assess persistent topological characteristics directly from data points with the evaluation of the geometrical information of the system and propose a way to measure the limit of quantitative methods and the necessity to seriously account pure empirical - observational methods when this limit is surpassed.

\section{THEORETICAL BACKGROUND}

\subsection{Conceptual basis}

Our systems analysis is based on two main concepts: the quantification of the system 's persistent entropy (Diaz Ochoa, 2020) and the analysis of the causal relationships in the system. The first complexity measure quantifies persistent structures in time series leading to individual system's behavior differing from the rest of the population. The second complexity measure helps to identify the interrelation of causal processes at different scales.

While the first complexity measure provides information about how the underlying mechanisms and theory can be extrapolated in a population, the second complexity measure delivers information about subtle phase changes of the observed system. By analyzing the system 's autonomy we are going beyond the mere modelling of the epistemic uncertainty ${ }^{8}$, since we open the door to the possibility that this uncertainty is not fortuitous or random (or is a systematic error of the observation) but is generated by the inherent autonomy of living processes. We focus our analysis on the use of integrated information using persistent homology.

\subsection{INTEGRATED INFORMATION}

The integrated information theory has become a relevant tool to measure the way how dynamical processes should be understood as a whole, considering that complex systems are essentially nonreducible. In this way systems with different microscopic degrees of connectivity are different as the causal processes accounted by fully connected systems, generating a complexity upper-bound by the total information of the connected and disconnected system. This theory, which has its origin in neurosciences, essentially measures quantifies multiple causal influences among elements of a System, such that it serves as a marker about how a nervous system integrates information (Koch, 2019), which is a fundamental notion defining consciousness. However, the same theory has been used as an alternative complexity measure useful to measure causal influences among elements in different complex system like physics, economics or biology, and which has been used to understand the evolution of complex organisms based on the relation between their ability to integrate information and their biological fitness (Joshi et al., 2013a).

\footnotetext{
${ }^{7}$ And how this network theory can (or cannot) be universalized, i.e. extrapolated to different complex systems

${ }^{8}$ https://towardsdatascience.com/probabilistic-neural-networks-for-breast-cancer-detection-2f1a6951e459
} 
Our rationale is to use this complexity measure to assess possible changes in causal paths that might be generated by changes in the intrinsic connectivity between system's internal states. This implies that any data analysis implicitly requires a condition of connectivity, i.e., the existence of a structure $\widehat{M}$ that integrates information and allows the computation of a trajectory $\Gamma$, such that $P(\widehat{M})=\Gamma$. The change in these causal paths is eventually generated from environmental factors: while stable mechanisms are associated to constant causal paths (low complexity), changes in the intrinsic connectivity leads to changes in the causal paths (and therefore a change in the measured complexity).

To this end we adapt the use of the measurement of the intrinsic cause power $\Phi$ to assess the degree of autonomy of the system. According to this theory, wholes / Coarse grained behavior cannot be simply reduced to interacting elements, delivering in this way a measure about the autonomy of the system (Marshall et al., 2017) (Koch, 2019). We adapt in this investigation the concept of integrated geometrical information $\Phi_{G}$ (Oizumi et al., 2016), considering all the possible connected and disconnected states. However, instead of analyzing the states as connected / disconnected (Marshall et al., 2017) we assume that we observe the whole state and evaluate $\Phi$ in different time periods, assuming that in such periods there are connected / disconnected internal states, which cannot be partially or fully accessed and which are encoded by the structure $\widehat{M}$.

From the geometrical integrated information theory, we consider the following fundamental postulates:

Postulate 1: The strength of the influences is quantified by a minimized difference between fully connected and disconnected models.

Postulate 2: A disconnected model system satisfies a Markov condition

Postulate 3: The difference between a connected and a disconnected model is measured by a $\mathrm{KL}$ divergence.

According to these postulates, and following the proof provided by Oizumi et al. (Oizumi et al., 2016)

$$
\Phi_{G}=\min _{q} D_{K L}[p(X, Y)][q(X, Y)]
$$

where $X=\left\{x_{1}, x_{2}, \cdots\right\}$ and $Y=\left\{y_{1}, y_{2}, \cdots\right\}$ are the past and present states of the system, $p$ is the joint probability function of connected states, and $q$ is the joint probability function of disconnected states, and $D_{K L}$ is the Kulback-Leiber entropy, defined as $D_{K L}[p(X, Y)][q(X, Y)]=$ $\sum_{X, Y} p(X, Y) \log \frac{p(X, Y)}{q(X, Y)}$.

Accordingly, $\Phi_{G}$ is upper bounded by the mutual information $I\left(x_{t^{\prime}}, x_{t}\right)$, such that $0 \leq \Phi_{G} \leq$ $I\left(x_{t^{\prime}}, x_{t}\right)$. In this way $\Phi_{G}$ fulfills the conditions required for the definition of integrated information. Therefore, we have different scenarios

- Low information loss by mechanistic systems

- Information loss in case that the system behaves in an autonomous way

- Fully information loss in case of fully disconnected and aleatory ${ }^{9}$ systems

\footnotetext{
${ }^{9}$ Aleatory implies fully causal disconnectedness. Stochastic uniform distributions could show a causal behavior, assuming that inherent mechanisms lead uniform distributed trajectories
} 
In the case of dynamic systems, this joint probability function is characterized by the system's trajectories in the phase space $\Gamma$, such that eq. 1 describes the distance between the phase space generated by connected and less connected systems.

Considering that the trajectories are computed based on intrinsic information processing based on interconnected structures $\widehat{M}$, such that the computation of a trajectory can be defined as $p(\widehat{M})=\Gamma$, then after introducing this expression into equation 1 , and based on the result of Oizumi el al. (Oizumi et al., 2016) we obtain

$$
\Phi_{G}=\min _{q} D_{K L}\left[p\left(\widehat{M}_{\tau}\right)\right]\left[p\left(\widehat{M}_{\tau^{\prime}}\right)\right] \leq I\left(\widehat{M}_{\tau}, \widehat{M}_{\tau^{\prime}}\right)
$$

i.e., $\widehat{M}_{\tau}$ and $\widehat{M}_{\tau}$, can be two different structures, where $I\left(\widehat{M}_{\tau}, \widehat{M}_{\tau}\right)$ represents the mutual information. According to this definition, the divergence of $\Phi_{G}$ implies a divergence of microstates, which simultaneously measures the degree of autonomy of the system, because the probability distribution depending on $\widehat{M}_{\tau}, p\left(\widehat{M}_{\tau}\right)$, behaves in a different way as the second probability distribution, $p\left(\widehat{M}_{\tau^{\prime}}\right)$, depending on the response to the environment, i.e. $p\left(\widehat{M}_{\tau}\right)$ is equivalent to $p\left(x_{t^{\prime}}, x_{t}\right)$ and $p\left(\widehat{M}_{\tau^{\prime}}\right)$ is equivalent to $q\left(x_{t^{\prime}}, x_{t}\right)$. Observe that we are comparing the system in two different periods of time, which is essentially a difference respect the original method in the eq. 1.

This approach is helpful to track possible inherent changes in $\widehat{M}$, and this can only be made assuming adaption / evolution of the system. Thus, instead analyzing different models leading to autonomous systems (Oizumi et al., 2016) we are analyzing the coarse grained state of the system with an underlying structure $\widehat{M}$. In this analysis we assume that we do not have information about $\widehat{M}$; but the analysis of the trajectory $\Gamma$ provides information about the preservation of causal rules, and therefore about the autonomy of the system and the possibility to derive $\widehat{M}$ as the underlying causal structure of $\Gamma$ when $\Phi_{G} \rightarrow 0$.

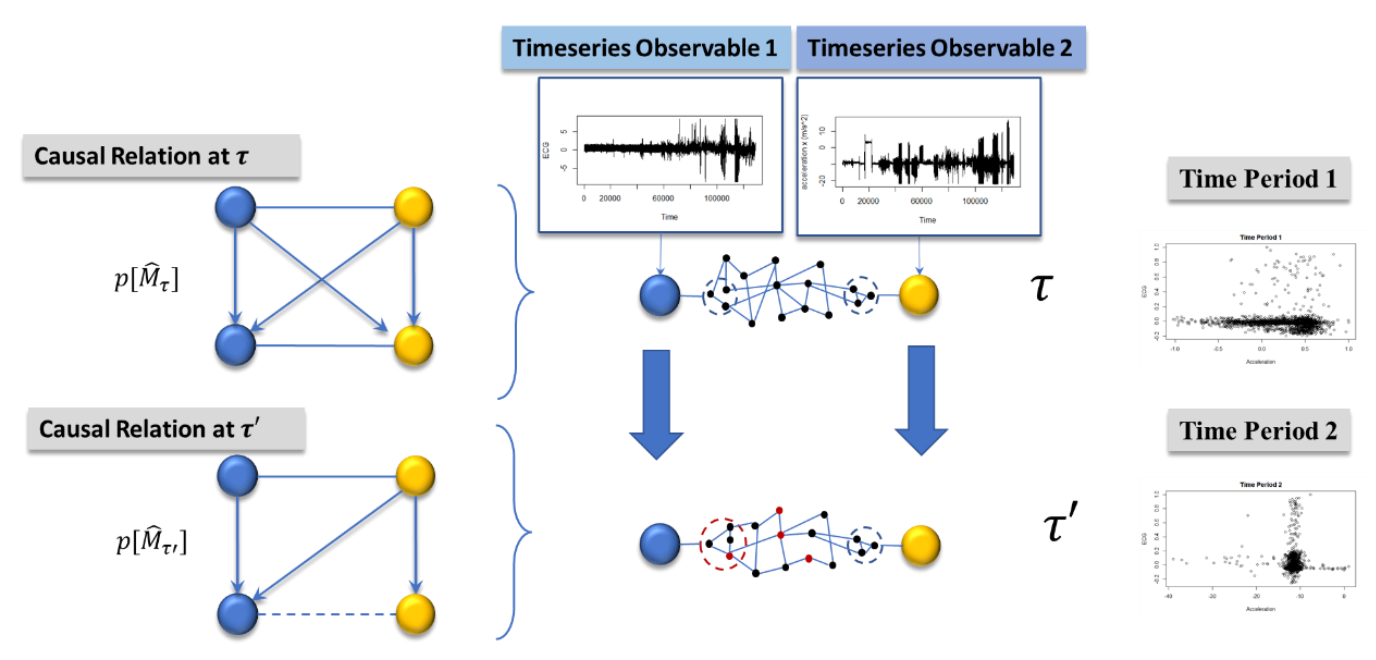

Figure 1 Causal relationship for a system with changing interactions (internal as well as external). Instead to defining an interactome ${ }^{10}$, leading to a comprehensive and complete model, we aim here to analyze from the data potential changes in the underlying interactions or the coarse graining required in the definition of the final observations

${ }^{10}$ https://en.wikipedia.org/wiki/Interactome 
Of course, we cannot make a detailed analysis between connected and disconnected systems based only on $\Gamma$. But we can analyze the structure of $\Gamma$ by estimating the difference of persistence bars $L[\Gamma]$ inside different time periods, as has been demonstrated in previous works ${ }^{11}$ (Diaz Ochoa, 2020).

To this end we assume that $\Gamma$ owns a topology that reflects the periodic behavior of a signal with Euler characteristics, this means $\Gamma$ owns a function $g$ with a compact subset of $\mathbb{R}^{D}$ and $d_{\bar{\Gamma}^{k}}: \mathbb{R}^{D} \rightarrow \mathbb{R}$ the distance function of $\bar{\Gamma}^{k}$. The function $L=\left\{\delta: d_{\bar{\Gamma}^{k}}(\delta) \leq \varepsilon\right\}$ represents the set of persistent bars $\delta_{\varepsilon}$ containing the length of the topological feature extracted from the periodic behavior of the signal. In this context, a barcode is the persistence analogue of a Betti number. Recall that the kth Betti number of a complex, acts as a coarse numerical measure of the topological feature $H_{k}$. Key topological features $H_{k}$ include zero (connected points) and first order topology (loops) (Diaz Ochoa, 2020). In this way, we essentially estimate a hierarchical grouping of topological characteristics of higher order leading to a type of invariants represented by bar codes (Pun et al., 2018). The use of this methodology is thus a way to track subtle changes in the characteristic trajectories in different time periods, as is presented in figure 1.

We compute the Kullback Leiber distance of $L[\Gamma]$, assuming that they are implicitly generated by systems with different connectivity, such that the equation 2 gets the following form

$$
\Phi_{G P}^{\prime}=\min D_{K L}\left[L\left[w_{\tau}\right] \| L\left[w_{\tau}\right]\right] \leq I\left(\Gamma_{\tau}, \Gamma_{\tau^{\prime}}\right)
$$

where $\Phi_{P} \sim \Phi_{G P}^{\prime}$ is the geometric integrated information, measured on the persistent topological characteristics of the trajectories of the single organisms; and according to these postulates, the measurement of the causal relation between underlying connectivity $\widehat{M}_{\tau}$ and $\widehat{M}_{\tau}$, can be mathematically accounted by the Kulback-Leiber divergence between the probability distributions of fully connected and disconnected systems ${ }^{12}$, i.e. by measuring the degree of independency between the topological characteristics in different time periods. The workflow of this computation is shown in Figure 2. All the computations were implemented using the TDA package in $\mathrm{R}^{13}$.

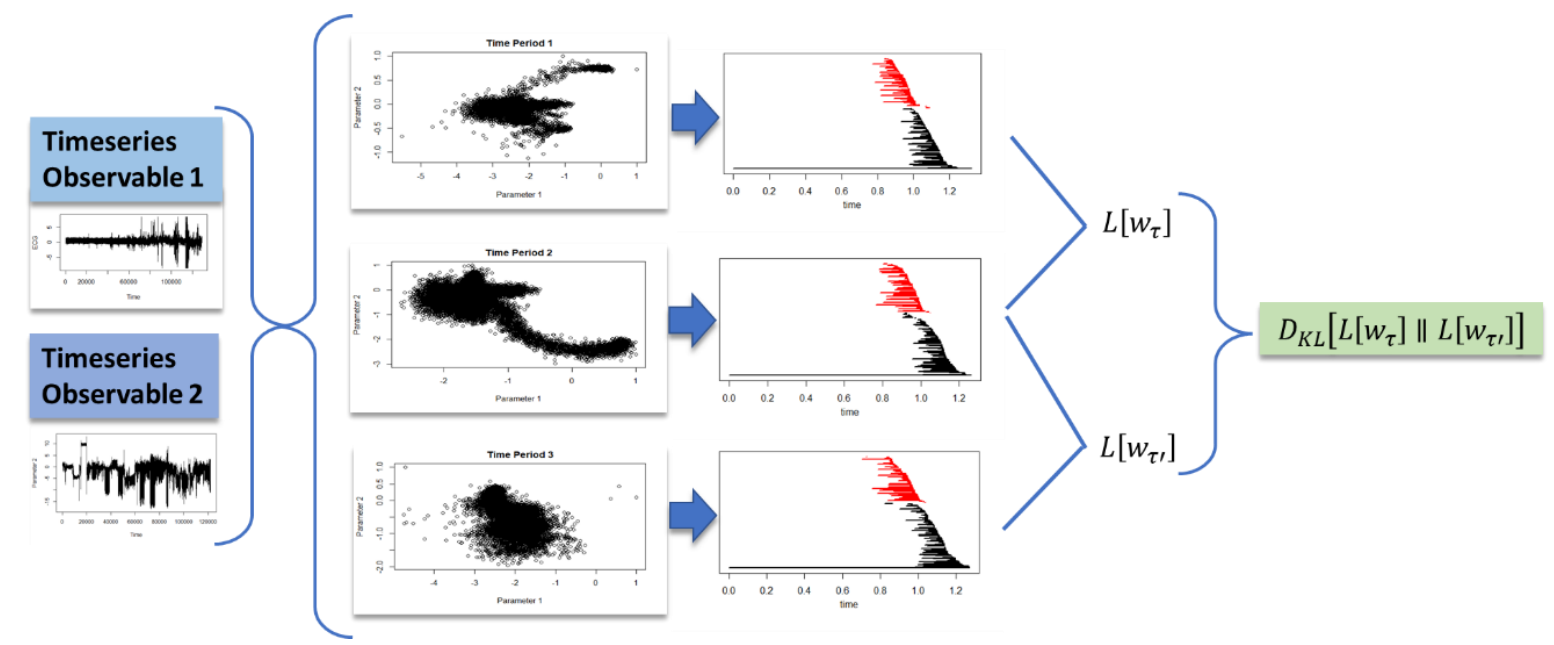

Figure 2 Workflow for the computation of $\boldsymbol{\Phi}_{G P}$ based on the computation of persistent bars obtained from time series, considering the construction of clouds of points form timeseries extracted in different time periods, the computation of persistence bars and the final computation of the Kallback-Leiber entropy for the estimation of $\boldsymbol{\Phi}_{G P}$.

\footnotetext{
${ }^{11}$ For the definition of the computation of persistence bars see the following publications

${ }^{12}$ Computed in this work using the Kullback Leiber Plugin in R: https://www.rdocumentation.org/packages/entropy/versions/1.3.1/topics/KL.plugin

${ }^{13}$ https://cran.uni-muenster.de/web/packages/TDA/vignettes/article.pdf
} 
In these evaluations is impossible to know if systems have a clear causal structure derived from a mechanistic causal structure $\widehat{M}$. However, systems exploring their environment in different ways should stablish different inherent causal relations to interpret this environment, such that the connectivity of the model changes accordingly. Based on these postulates, if there is a deviation in the measured probability distribution, as is evaluated with the workflow in figure 3 , then there is probably an intrinsic change in system (internal mechanisms as well as its interaction with the environment), impairing this way the observability of the system (Figure 2), i.e. the possibility to identify mechanisms interlinking different observables in a given system.

This situation is different to the mere concept of evolving networks since we are not only tracking a constant causal difference in a single system (dynamic and evolving system with dynamic interlinks), but also the interindividual variations, such that some individuals might evolve, while other systems own a stable inherent structure.

The tracking of the inter-species differences is thus a signal about the inherent autonomy of the systems. Therefore, the goal is not to model the uncertainty to get a complete model ${ }^{14}$, but discover how uncertainties push sometimes systems to behave in an autonomous way.

A.

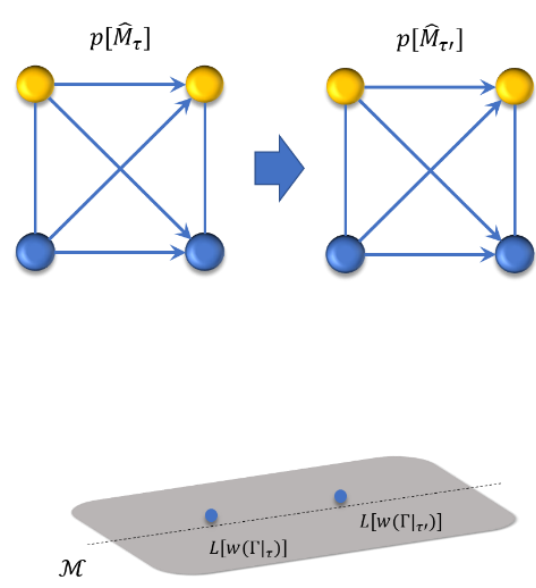

B.
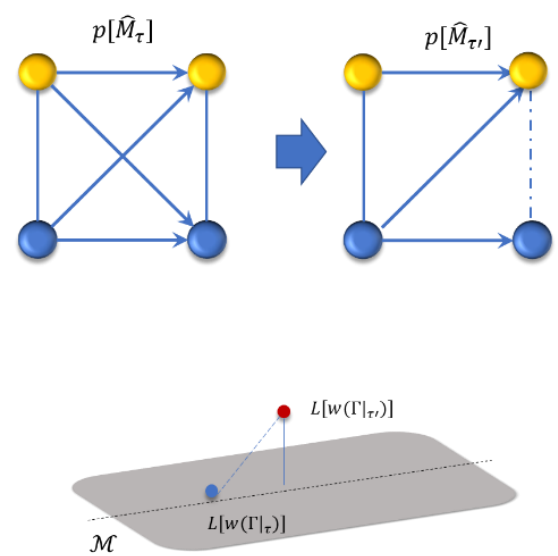

Figure 3 Effect of the change of internal causality on the system topology and consequent computation of persistence bars

According to these postulates, and the concept of mutual information ${ }^{15}$, the upper bound of the mutual information is the join information or join entropy ${ }^{16}$, which measures the uncertainty in the set of observations, and is defined as $H\left[\Gamma_{\tau}, \Gamma_{\tau^{\prime}}\right]=\sum_{w_{\tau}, w_{\tau^{\prime}}} P\left(\Gamma_{\tau}, \Gamma_{\tau^{\prime}}\right) \log \left(\Gamma_{\tau}, \Gamma_{\tau^{\prime}}\right)$, where $P\left(\Gamma_{\tau}, \Gamma_{\tau^{\prime}}\right)$ is the joint probability. Accordingly, the equation 3 takes the following form:

$$
0 \leq \Phi_{G P} \leq H\left[\Gamma_{\tau}, \Gamma_{\tau^{\prime}}\right]
$$

Such that, if $\Phi_{G P} \rightarrow 0$ then the connectivity remains invariant and constrained into a single manifold. Otherwise, the structure of the probability distribution diverges. Therefore, when $\Phi_{G P} \rightarrow H\left[\Gamma_{\tau}, \Gamma_{\tau^{\prime}}\right]$ it implies that the inferred causal relations tend to have the same degree of observed uncertainty, leading to extreme variable and eventually aleatory systems (see Figure 3 ). The interpretation of this expression and its practical application is provided in the next section.

\footnotetext{
${ }^{14}$ Which is the goal of several modern modelling technologies

${ }^{15}$ https://en.wikipedia.org/wiki/Mutual information

${ }^{16}$ https://en.wikipedia.org/wiki/Joint entropy
} 


\section{3 Ф-S DIAGRAM}

While the previous analysis aims to assess the degree of the internal connectivity between the elements of an observed system at a given scale, the estimation of the persistent entropy (Diaz Ochoa, 2020) ${ }^{17}$ helps to assess the relative inherent differences between individual organisms.

Thus, by using the measurement of the geometrical (and persistent) integrated information as well as the persistent entropy we can deploy a diagram to estimate the regions where different organisms probably behave in an individual and autonomous way.

The $\Phi$-S Diagram essentially solves the following questions: i) can the underlying mechanisms and theory be extrapolated from one to other organisms, or a whole population? and ii) are these mechanisms obeying constant causal relationships?

The positive answer to both questions is the basis for universal theories, as well as models that can be extrapolated to any other system or organism.

According to this representation, we define a vector $\Phi_{S}$ for each observed organism $k$ or system ordering the amount of integrated geometric information $\Phi_{G P}$ (causal analysis) and the mean amount of persistent entropy $S\left(\Gamma^{k l}\right)$, i.e. $S\left(\Gamma^{k}\right)=\frac{\sum_{k} S\left(\Gamma^{k l}\right)}{N_{k}}$, where $N_{k}$ is the total amount of other reference organisms. Thus, the $\Phi$-S Diagram for each organism $k$ is defined as.

$$
\boldsymbol{\Phi}_{S}{ }^{k}=\boldsymbol{\Phi}_{S}\left\{\Phi_{G P}\left(\Gamma^{k}\right), S\left(\Gamma^{k}\right)\right\}
$$

The representation of this vector and the distances measured on the main axes $\Phi_{G P}$ and $S\left(\Gamma^{k}\right)$, and the relative distance to low entropy values provides a map about observability / autonomy of the population. The interpretation of the diagram is presented in figure 3 , for systems behaving in a mechanistic way (Figure 4, i.) and in an autonomous or aleatory way (Figure 4, ii.)

i.

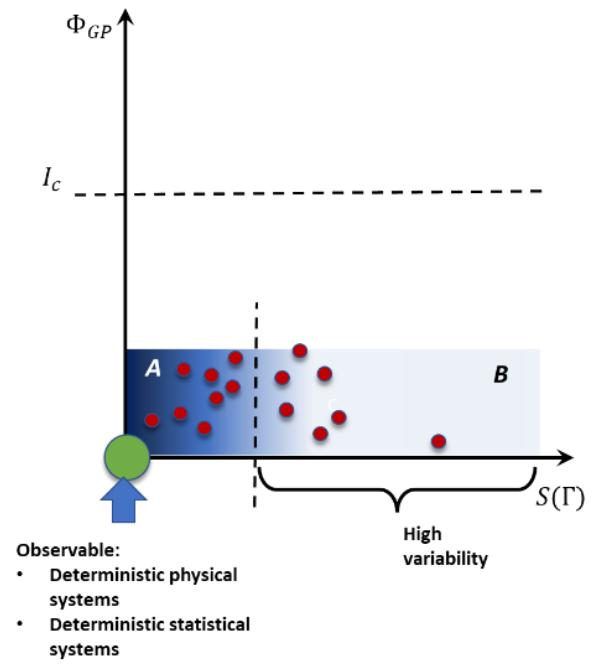

ii.

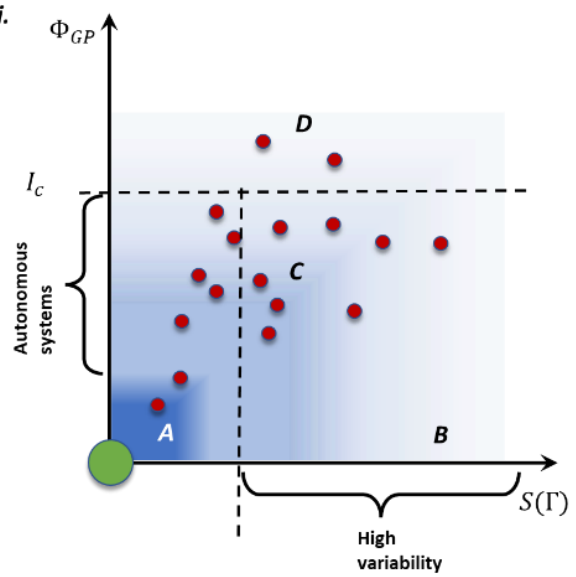

Figure 4 Interpretation of the $\Phi$-S Diagram based on the composition of the geometric integrated information $\boldsymbol{\Phi}_{G P}$ and the persistent entropy, for mechanistic (region A), mechanistic but high intersystem variability (region B), autonomous (region C) and aleatory systems (region D).

The shortest persistent bars in the diagram essentially represent noise and are therefore filtered in the final evaluation, such that any entropy measurement represents the relative grade of disorder of

${ }^{17}$ The detailed estimation of this entropy is provided in this article 
persistent patterns in the paths, and not the inherent noise of the system. The vector $\boldsymbol{\Phi}_{S}{ }^{k}$ represents the degree of disorder in persistent patterns from the measured paths, and while $S\left(\Gamma^{k}\right)$ represents the relative degree of disorder of these paths (degree of individual behavior), the second measure $\Phi_{G P}\left(\Gamma^{k}\right)$ represents the degree of autonomy.

The usability of the $\Phi$-S Diagram depends on an approximate definition of threshold values defining the three regions represented in Figure 5. Here we assume that the degree of autonomy corresponds to states changing between different regular individual mechanisms characterized by $\Phi_{S} \rightarrow 0$, and completely chaotic states characterized by $\Phi_{S} \gg 0$ (aleatory system). To better interpret this diagram, we set the following criteria to recognize if the system is either mechanistic, autonomous, or aleatory:

i. $\quad$ Considering the probability $w$ that a half of the persistent patterns are mutually divergent, then by replacing this probability in the definition of the entropy we obtain $S\left(\Gamma^{k l}\right) \approx 0.67$, which is an approximation to define a threshold defining whether the system 's states mutually diverge or not: below this value the persistent patterns tend to be non-divergent (similar mechanisms across different systems, region A in figure 4); otherwise, patterns are divergent, representing high intersystem variability (region $B$, figure 4 )

ii. If $\Phi_{G P}$ lies below the mean value of the join entropy, and the mean joint entropy is relatively low then the system behaves in a mechanistic way; otherwise, the system has several phase transitions, is probably autonomous depending on the information it processes (region $\mathrm{C}$, figure 4), or is aleatory (region $D$, figure 4 ).

Of course, we don't value the autonomy of the system in the same way as the integrated information, since in principle we do not have access to the system's structure. However, indirectly $\Phi_{S}$ traces the probably coarse-grained autonomy of the system: if $0<\Phi_{G P}<H\left[\Gamma_{\tau}, \Gamma_{\tau^{\prime}}\right]$, then this indicates that there are phase changes in the trajectories that do not necessarily originate from an autonomous behavior; but a simultaneous interindividual variability implies that these phase changes are individual and thus probably coarse-grained autonomous.

With these two threshold values is then possible to fix the space where simple causal - mechanistic principles can be established. In general, when $\left\|\boldsymbol{\Phi}_{S}\right\| \rightarrow 0$ then there is a probability to find stable inherent causal structures responsible of mechanisms leading to the identification of a model. Otherwise, a probable non-observability (rooted on a coarse-grained causality) can be stablished.

Thus, this diagram is like navigating with a map, helping to assess the degree of observability of single organisms regarding all the population. In both cases, if both entropies tend to be zero, then the system is probably observable and can be described using mathematical models. Once this is guarantee, then assessment about whether the inherent mechanisms of the system can be represented by black or white/grey box models, or if only an empirical approach is the most appropriate way to understand the system.

\section{RESULtS}

In this first test we aim to test this methodology on a deterministic dataset consisting of the combination of the semi-periodic timeseries ${ }^{18}$ that are permuted to synthesize different individual systems generating their own time series. Here we expect to obtain a deterministic model leading to

${ }^{18}$ https://archive.ics.uci.edu/ml/datasets/Pseudo+Periodic+Synthetic+Time+Series 
features representing some degree of observability, i.e., model and parameter identification; simultaneously we also expect high interindividual variability, since the original time series are permuted to generate each individual system. This should be characterized by a $\boldsymbol{\Phi}_{S}{ }^{k}$ value representing both a high degree of causality and relatively low persistence of defects.

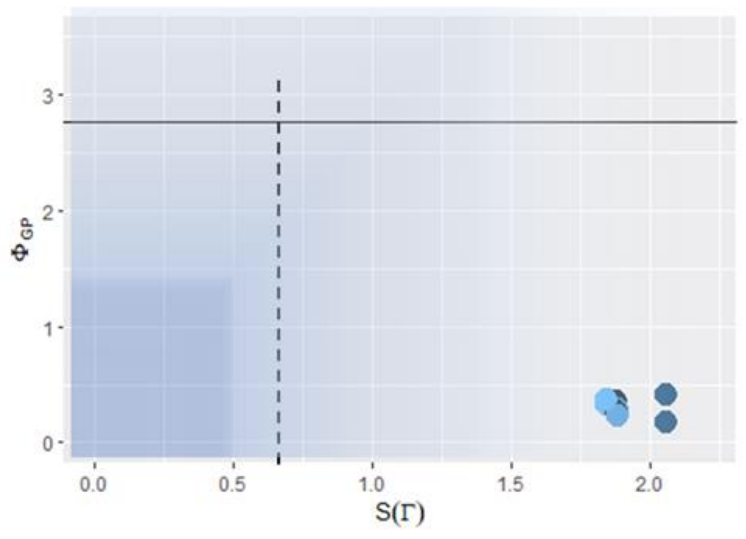

Figure 5 Ф-S Diagram for different deterministic systems

The analysis for three different periods is deployed in Figure 5, where the distribution of $\left[\boldsymbol{\Phi}_{S}{ }^{k}\right]_{i}$ for each individual $i$, is represented as a distribution (Fig. $5 \mathrm{~A}$ ) and as a double boxplot (Fig. 5-B). From the computation of $\boldsymbol{\Phi}_{S}{ }^{k}$ we have found, as expected, that almost for all the systems $\Phi_{G}<0.5$ (besides one outlier), i.e., for all the system there is a high probability to share same intrinsic causal rules (low autonomy). Simultaneously we observe a high inter-individual variability, i.e., $S\left(\Gamma^{k l}\right)>0$. This implies that there are persistent characteristics that differ between individual systems.

In our next example we use the $\mathrm{M}$-Health data $^{19}$ to analyze the correlation between cardiac response to exercise. In this database individuals were provided wearables to measure acceleration in three axes and other physiological parameters like ECG to measure the response of the participants to sport. The aim of this data is thus to measure the response to sport in rather real situations and outside from the labor in order to have realistic data about the response to exercise. The heart rate regulation system was conceptualized as a complex network, with non-linear feedforward and feedback inputs. This system exhibits chaotic and non-linear dynamics, due to interactions between physiological oscillators, functional state changes, and noise (Dimitriev et al., 2020)(Voss et al., 2009) .

Despite this non-linear character of the cardiac response, we assume that it should be possible to generate a simple $A \leftrightarrow B$ model based on the correlation between both states, i.e. $A(x, y, z)=$ $f_{1}(B)$, and $B=f_{2}(A(x, y, z))$ where $A(x, y, z)$ is the acceleration measured in three different axes, and $B$ is the heart response measured using an electrocardiogram (figure $6 \mathrm{~A}$ ). This simple model implies that for different individuals in a small population there is a high probability to find similar causal relationships in the response to exercise, i.e., there is a simple and mechanistic causal relation between the hearth response and physical exercise. Since we are analyzing along the tree axis, we can first analyze $\Phi_{G}$ as a 3D distribution ${ }^{20}$.

\footnotetext{
${ }^{19}$ https://archive.ics.uci.edu/ml/datasets/MHEALTH+Dataset

${ }^{20}$ To this end we used the sthda package in R: http://www.sthda.com/english/wiki/a-complete-guide-to-3dvisualization-device-system-in-r-r-software-and-data-visualization
} 
A.

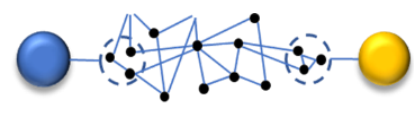

A

B

B.
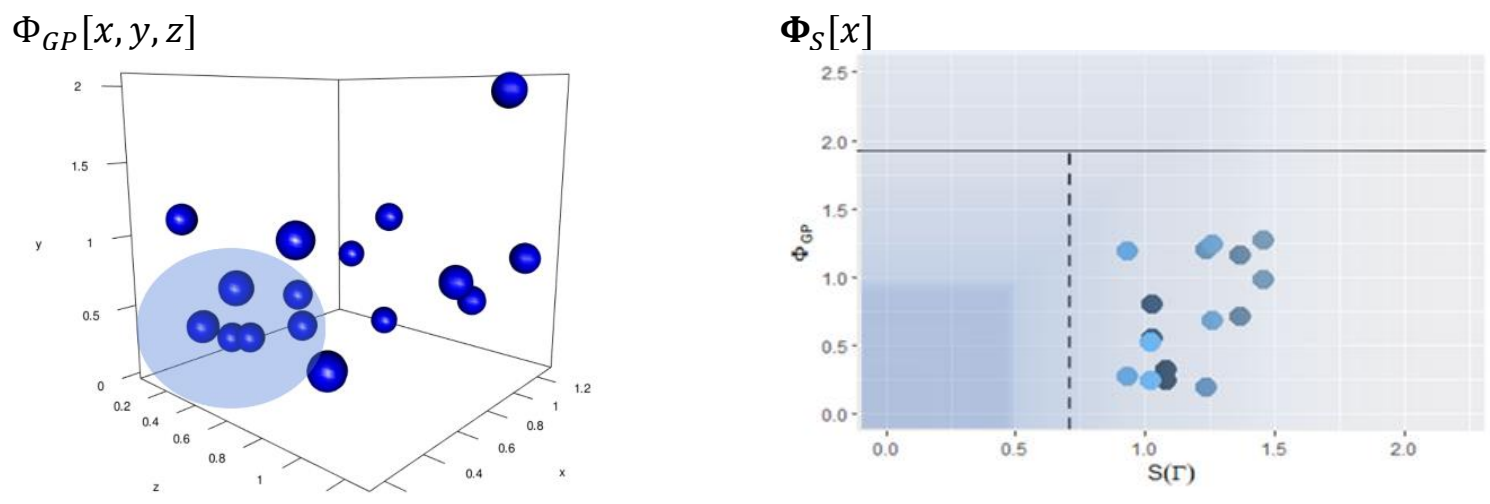

$\boldsymbol{\Phi}_{S}[y]$

$\boldsymbol{\Phi}_{S}[z]$
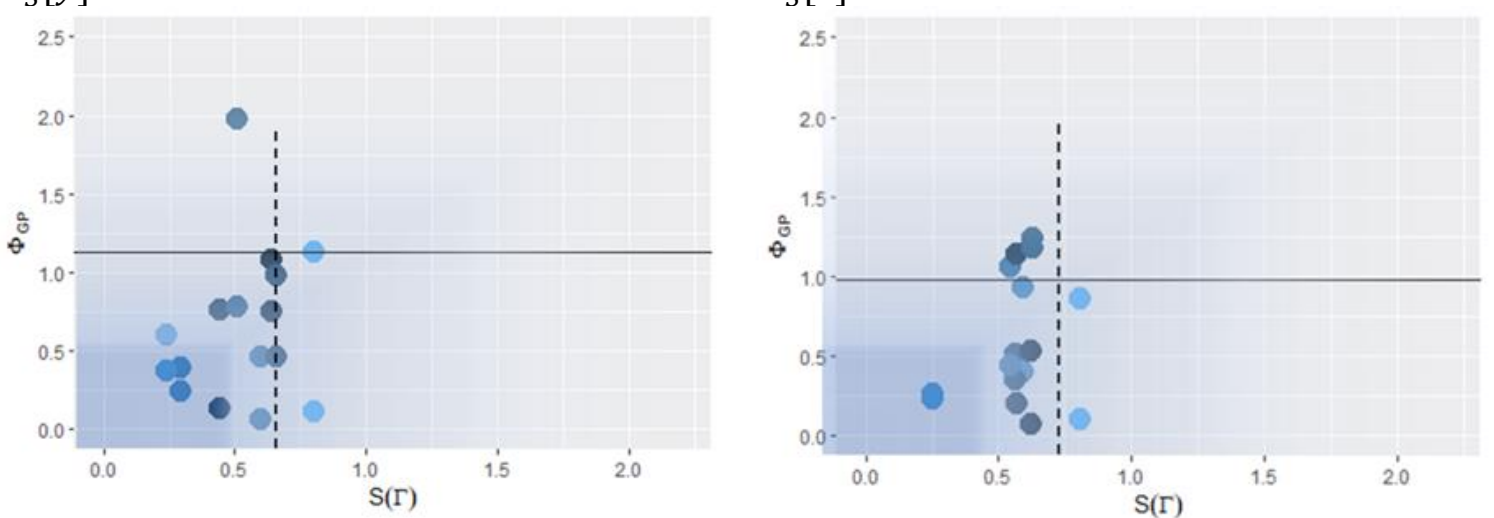

Figure 6 Analysis of the cardiac response to sport assuming tha the physical excersise (meassured as acceleration A) has a correlation to the cardiac response $B$. The possibility to induce/deduce a model requires a low causal entropy $\Phi_{G}$; however, a first inspection of this entropy along the three axes demonstrates that only few individuals have this low causal entropy (Figure B, 3 D diagram). The $\Phi$-S Diagram for each acceleration axis is also deployed.

The 3D representation is helpful to understand the meaning of the present analysis: when this complexity measure "condenses" inside the blue bubble, then the causal relations are stable, which means that this system cis feasible to be modelled, i.e., that the recognized patterns can for instance be used as features for inductive (Al) models. Otherwise, there is an "informational evaporation", close to the condensation region the system is probably autonomous. But for larger "informational evaporation" the system is either highly autonomous or aleatory.

Thus, we have found out that de facto a coupling between cardiac response and physical exercise close to the blue region and confirm the feasibility for the identification of both inductive and deductive models, or a combination of both methods. But there are also strong deviations that suggest that this coupling is much more complex and less correlated. The analysis of $\boldsymbol{\Phi}_{S}{ }^{k}$ on the $\mathrm{x}$ axis reflect different movement patterns in some individuals, implying an autonomous response to exercise. There is also a high interindividual variability, i.e., only for few individuals a kind of universal model could correctly represent the response to sport. 
Since only few individuals fall into the blue square reserved for almost mechanistic systems, we conclude that any modelling effort will be confronted to a high degree of uncertainty, and that a common calibrated model for all individuals is feasible (in particular for a model only on the $y$ axis) but will not accurate enough for model all the individuals.

\section{DISCUSSION}

Many scholars have recently observed that we are currently amid a Copernican revolution in biology: like the celestial mechanics, where the trajectory of the planets has been correctly described using basic physical laws represented in an elegant mathematical way, biologically living systems could soon also be represented with simple laws and consistent mathematical concepts. And taking into account a reductionist perspective, such biological laws should also be derived from basic physical laws (Kesić, 2016). The game or life is the perfect example about how the combination of deterministic laws and chance, resembling random mutations and natural selection, could lead into cellular automata models mimicking simple living forms (Berto and Tagliabue, 2021) ${ }^{21}$. In this way, the cojoin of two mean characteristics, namely dependence and autonomy, are mediated between extreme forms of dualism, which reject the micro-dependence of some entities, and reductionism, which rejects macroautonomy ${ }^{22}$ (O'Connor, 2020), a characteristic observed in several complex systems like complex networks.

Such notion of emergence is the corner stone for the formulation of systemic approaches leading to mathematical descriptions in different scales of molecules and pathways as well as whole populations of organisms, leading to an understanding about how living organisms metabolize, replicate and form populations and ecologies ${ }^{23}$. From these simple laws is then possible to derive a systemic theory aiming to model/describe any bio-medical system with relatively high precision, allowing to perform synthetic biology as well as modify (or hack) biological processes. These ideas have become a hope in order to siege over diseases and death, innovate and optimize production processes in biotechnology and modify biological processes and even help to bring humans into other planets (Verseux et al., 2016).

However, these ideas assume that causal relationships as well as a relative uniformity between different organisms within a tolerance range can be identified, which leads to the identification of mechanisms or correlations. On the other hand, organisms are known to sometimes exhibit great biological variability, perhaps originating not simply in the stochastics of the system, but also in their inherent macro autonomy and ability to integrate information linked to the evolution and complexity of organisms (Joshi et al., 2013a). For example, and contrary to the generally accepted notion of the prevalence of blind and random mutations that act as the engine of evolution, it has recently been discovered that mutations in Arabidopsis thaliana are biased and that genes protect relevant parts of the genome, and that mutations occur less frequently in functionally restricted regions.(Monroe et al., 2022).

Mechanistic approaches had also inspired concepts that deny complex forms of autonomy in apparent simple organisms, leading to perceptions such as viewing insects as unconscious and robotic, with little

\footnotetext{
${ }^{21}$ https://plato.stanford.edu/entries/cellular-automata/\#CAEmer

22 https://plato.stanford.edu/entries/properties-emergent/

${ }_{23}$ This concept is influent in several communities, not only in biology, biasing in some cases the way how living beings are considered and studied, in some cases reducing them to mere mechanisms
} 
more emotional depth than lumps of stone ${ }^{24}$. Only recently has there been a different focus on complex biological systems ${ }^{25}$ who have even recognized the possibility that honeybees have behaviors that could reflect mood states (Bateson et al., 2011).

All these examples suggest that living systems are not blind on any scale. A reductionist as well as a systemic approach (leading into a synthetic organism) could in some cases be a limited perspective, either for an entire population or for individual individuals, and identification of complex forms of autonomy is necessary before models are formulated and validated.

Using techniques inspired from the concepts of the integrated information as a way to measure the complexity of a system in relation to its ability to integrate information (Koch, 2020) (Oizumi et al., 2016)., we have develop a novel methodology based on persistent topology to analyze the patterns in time series of states that would be potentially coupled through a model. This method helps to precisely assess if a model, and in general an objective systemic reduction of a system, is feasible or not by scanning the causal relations in the response of a system. This scan is then deployed in what we have defined as a $\Phi-S$ Diagram, which is essentially the combination of two complexity measures: the observed integrated information from the empirical measurements and the persistent entropy.

This method assumes the fact that systems behave as a whole and as autonomous observers evaluating their environment regardless the system's scale, limiting our ability to identify well-defined mechanisms. Thus, the $\Phi-S$ Diagram is like a Ptolemais map, i.e., is a cartography of organisms that can be either placed in a known world due to its mechanistic character (small corner in the diagram), vs. organisms with larger entropy generated by their inherent autonomy belonging to a larger and less explored space in the map (limited-observability).

Persistent entropy and the autonomy of the system imply limitations that ultimately could not be overcome with mere engineering, not only in terms of a possible limitation in the way we objectively describe the world, considering that other organisms also interpret the world from a subjective perspective, but also how possible ethical problems might arise, by trying to derive objective observations. This could have serious implications for the way machine learning and artificial intelligence are used to model biological systems. For example, some argue that machine learning could be used to overcome the current scalability limitations of mechanistic modeling, while mechanistic models of machine learning algorithms could be used both as transient inputs and as validation frameworks (Baker et al., 2018); in this framework our methodology aims to evaluate the feasibility of this marriage and the fact that systems eventually go beyond the expected mechanistic perspective. Specifically, the vast majority of machine learning applications is aimed at supporting statistical or correlation studies that bypass the need for causality and focus exclusively on prediction (Baker et al., 2018).

With the $\Phi$-S Diagram is thus possible to comprehend and track this causality before starting any modelling effort: at some point there is a factual boundary where a correlation-based model is not enough to describe a system, while a persistent empirical approach is the only way to understand the actual state of a system, which is an important fact not only for biology but also for medicine in evaluating how mechanistic reactions should be studied, taking into account that these causal relationships lead to medical outcomes (Reiss and Ankeny, 2016).

${ }^{24}$ https://www.bbc.com/future/article/20211126-why-insects-are-more-sensitive-than-they-seem

${ }^{25} \mathrm{~A}$ feature belonging to non-trivial autonomous systems 


\section{CONCLUSIONS}

In this work we have reported on a novel method based on persistent homology to recognize patterns in dynamic trajectories helping to identify causal mechanisms as well as interindividual variability in dynamic trajectories, and in this way track non-trivial autonomous response/behavior patterns. Therefore, this method is essentially a kind of unsupervised machine-learning method that relies on the fundamental topological characteristics of system trajectories. This method leads to a novel complexity measurement, defined here as a $\Phi$-S Diagram, that helps to determine the observability of a given system, i.e., the feasibility to represent this system either with a deductive or an inductive model. Thus, this method helps to estimate the degree of inherent uncertainty in the system, and the feasibility to represent it in a consistent mathematical way.

We deduced the theoretical background, the way to interpret the results delivered by the $\Phi$-S Diagram and demonstrated the use of this methodology in two datasets, one based on pure-deterministic data and the second one based on physiological data captured from patients performing physical exercise. The obtained results help thus to estimate if for certain individuals a common morel is enough to represent its physiological behavior, or if individualized methods or an empirical approach is required.

The presented results are preliminary, and additional tests and analysis is required, for instance the extension of this methodology for the efficient analysis of high dimensional datasets. Furthermore, additional work is required to match the presented theory with the foundations and theoretical background of the theoretical biology/systems biology as well as for its application in a productive framework, for instance in the exact assessment about how reliable is to make decisions based on models, as well as in establishing cartographic methods in the analysis of the degree of uncertainty of complex systems.

\section{COMPETING INTEREST}

The author declares no competing interests.

\section{BIBLIOGRAPHY}

Alon, U. (2006). An Introduction to Systems Biology: Design Principles of Biological Circuits (Boca Raton, FL: Chapman \& Hall/Crc Mathematical \& Computational Biology).

Baker, R.E., Peña, J.-M., Jayamohan, J., and Jérusalem, A. (2018). Mechanistic models versus machine learning, a fight worth fighting for the biological community? Biol. Lett. 14, 20170660.

Bateson, M., Desire, S., Gartside, S.E., and Wright, G.A. (2011). Agitated Honeybees Exhibit Pessimistic Cognitive Biases. Curr. Biol. 21, 1070-1073.

Berto, F., and Tagliabue, J. (2021). Cellular Automata. In The Stanford Encyclopedia of Philosophy, E.N. Zalta, ed. (Metaphysics Research Lab, Stanford University), p.

Boogerd, F., Bruggeman, F.J., Hofmeyr, J.-H.S., and Westerhoff, H.V. (2007). Systems Biology: Philosophical Foundations (Elsevier). 
Diaz Ochoa, J.G. (2020). Observability of Complex Systems by Means of Relative Distances Between Homological Groups. Front. Phys. 8, 503.

Dimitriev, D., Saperova, E.V., Dimitriev, A., and Karpenko, Y. (2020). Recurrence Quantification Analysis of Heart Rate During Mental Arithmetic Stress in Young Females. Front. Physiol. 11, 40.

Ellis, G.F.R., and Kopel, J. (2019). The Dynamical Emergence of Biology From Physics: Branching Causation via Biomolecules. Front. Physiol. 9, 1966.

Green, S. (2021). Philosophy of Systems and Synthetic Biology. In The Stanford Encyclopedia of Philosophy, E.N. Zalta, ed. (Metaphysics Research Lab, Stanford University), p.

Joshi, N.J., Tononi, G., and Koch, C. (2013a). The Minimal Complexity of Adapting Agents Increases with Fitness. PLoS Comput. Biol. 9, e1003111.

Kesić, S. (2016). Systems biology, emergence and antireductionism. Saudi J. Biol. Sci. 23, 584-591.

Koch, C. (2019). The Feeling of Life Itself: Why Consciousness Is Widespread but Can't Be Computed (Cambridge, MA: The MIT Press).

Koch, C. (2020). The Feeling of Life Itself: Why Consciousness Is Widespread but Can't Be Computed (Cambridge, MA: The MIT Press).

Kostić, D., Hilgetag, C.C., and Tittgemeyer, M. (2020). Unifying the essential concepts of biological networks: biological insights and philosophical foundations. Philos. Trans. R. Soc. B Biol. Sci. 375, 20190314.

Marshall, W., Kim, H., Walker, S.I., Tononi, G., and Albantakis, L. (2017). How causal analysis can reveal autonomy in models of biological systems. Philos. Trans. R. Soc. Math. Phys. Eng. Sci. 375, 20160358.

Monroe, J.G., Srikant, T., Carbonell-Bejerano, P., Becker, C., Lensink, M., Exposito-Alonso, M., Klein, M., Hildebrandt, J., Neumann, M., Kliebenstein, D., et al. (2022). Mutation bias reflects natural selection in Arabidopsis thaliana. Nature 1-5.

O'Connor, T. (2020). Emergent Properties. In The Stanford Encyclopedia of Philosophy, E.N. Zalta, ed. (Metaphysics Research Lab, Stanford University), p.

Oizumi, M., Tsuchiya, N., and Amari, S. (2016). Unified framework for information integration based on information geometry. Proc. Natl. Acad. Sci. U. S. A. 113, 14817-14822.

Pun, C.S., Xia, K., and Lee, S.X. (2018). Persistent-Homology-based Machine Learning and its Applications -- A Survey. ArXiv181100252 Math.

Ravetz, J. (2003). Models as metaphors. In Public Participation in Sustainability Science: A Handbook, B. Kasemir, C.C. Jaeger, J. Jäger, and M.T. Gardner, eds. (Cambridge: Cambridge University Press), pp. $62-78$.

Reiss, J., and Ankeny, R.A. (2016). Philosophy of Medicine. In The Stanford Encyclopedia of Philosophy, E.N. Zalta, ed. (Metaphysics Research Lab, Stanford University), p.

Sacks, O., Métraux, A., and Schmidt, R. (1991). Awakenings - Zeit des Erwachens: Das Buch zum Film (Reinbek bei Hamburg: Rowohlt Taschenbuch). 
Verseux, C., Paulino Lima, I.G., Baqué, M., Billi, D., and Rothschild, L. (2016). Synthetic Biology for Space Exploration: Promises and Societal Implications. pp. 73-100.

Voss, A., Schulz, S., Schroeder, R., Baumert, M., and Caminal, P. (2009). Methods derived from nonlinear dynamics for analysing heart rate variability. Philos. Trans. R. Soc. Math. Phys. Eng. Sci. 367, 277-296.

Walker, S.I. (2019). The new physics needed to probe the origins of life. Nature 569, 36-38.

Zitnik, M., Nguyen, F., Wang, B., Leskovec, J., Goldenberg, A., and Hoffman, M.M. (2019). Machine learning for integrating data in biology and medicine: Principles, practice, and opportunities. Inf. Fusion 50, 71-91. 